\title{
Visual evoked responses in multiple sclerosis: comparison of two methods for pattern reversal
}

\author{
B E N G T Y. N I L S S O N \\ From the Department of Clinical Neurophysiology, Södersjukhuset, Stockholm, Sweden
}

SUMMARY Visual evoked responses have been elicited by reversal of a black and white checkerboard pattern and also by a display of light-emitting diodes (LED) generating a reversal of a pattern of illuminated red circular areas. In a control group the LED display produced responses of lower amplitude and shorter latency compared to responses to checkerboard stimulation. In patients with a definite or probable diagnosis of multiple sclerosis the stimulators were equally effective in evoking pathological responses; in patients with a possible but less certain diagnosis abnormal responses were found in a higher percentage when the LED stimulus was used. Differences in stimulus field size and in stimulus colour are discussed as possible reasons for this result.

Although the visual evoked response (VER) to flash stimulation may be changed pathologically in multiple sclerosis (Feinsod and Hoyt, 1975; Paty et al., 1976), the diagnostic value of VER in demyelinating disease was considerably enhanced when Halliday et al. (1972, 1973) demonstrated that the major positive wave of the response evoked by pattern reversal stimulation is delayed in a high percentage of patients with retrobulbar neuritis and multiple sclerosis. The original method used by Halliday involves the backprojection of a checkerboard pattern on to a semitranslucent screen via a rotatable mirror, and reversing is accomplished by sideways movements of one square width. Results with this method have been reported subsequently by several groups (Asselman et al., 1975; Regan et al., 1976; Chain et al., 1977; Matthews et al., 1977). The checkerboard (Chb) pattern can also be presented on a television monitor (Bornstein, 1975; Arden et al., 1977; Hennerici et al., 1977). Details of stimulation procedure and stimulus properties have varied a little, but these reports share the finding of a high frequency of pathological responses in patients where the diagnosis of multiple sclerosis has been very probable, while the rate of abnormalities generally is rather low in patients where the diagnosis is considered as possible but less certain multiple sclerosis.

\footnotetext{
Address for reprint requests: Dr Bengt Y. Nilsson, Department of Clinical Neurophysiology, Södersjukhuset, S-100 64 Stockholm, Sweden.

Accepted 1 February 1978
}

Evans et al. (1974) described a simple method for pattern reversal stimulation which made use of a display of two identical sets of light-emitting diodes (LED) that could be activated alternately. The clinical usefulness of this method has been reported preliminarily (Purves and Low, 1976; Bynke et al., 1977; Nilsson, 1977). The present report deals with a direct comparison of results obtained with this LED stimulator and responses to a back-projected Chb pattern in a control group and in a series of multiple sclerosis patients.

\section{Methods}

The LED pattern reversal stimulator was built according to the description of Evans et al. (1974). It consisted of 64 light-emitting diodes of red colour (wavelength $650 \mathrm{~nm}$ ) and round shape (Litronix RL 5054-2), symmetrically arranged in eight rows and linked together in two series so that every second diode was lit simultaneously. The current is manually or automatically switched between the two diode chains, thus giving an appearance of a pattern reversal. The checkerboard pattern was produced by a commercially available device (Digitimer D110), which includes a slide projector and a rotatable mirror. The physical properties of the two patterns are summarised in Table 1. The main differences are:

Pattern colour Red light-emitting diodes were chosen because they had the maximal available brightness. The checkerboard pattern was black and white. 
Table 1 Physical properties of pattern composed of light-emitting diodes (LED) and checkerboard pattern (Chb) produced by slide projection

\begin{tabular}{lll}
\hline & $L E D$ & $C h b$ \\
\hline Pattern colour & red/black & white/black \\
Pattern element shape & circular areas & squares \\
Pattern element size & $20^{\prime}$ & $23^{\prime}$ \\
& $($ diam. $5 \mathrm{~mm})$ & $\left(6.7 \times 6.7 \mathrm{~mm}^{2}\right)$ \\
Total field size & $3.9^{\circ}$ & $8.8^{\circ}$ \\
& $\left(58 \times 58 \mathrm{~mm}^{2}\right)$ & $\left(154 \times 154 \mathrm{~mm}^{2}\right)$ \\
Viewing distance & $0.85 \mathrm{~m}$ & $1.0 \mathrm{~m}$ \\
Luminance & & $1000-1200 \mathrm{~cd} / \mathrm{m}^{2}$ \\
light parts & $325 \mathrm{~cd} / \mathrm{m}^{2}$ & $12-16 \mathrm{~cd} / \mathrm{m}^{2}$ \\
dark parts & $0.6 \mathrm{~cd} / \mathrm{m}^{2}$ & $50 / 50 \%$ \\
Illuminated/dark area & $19 / 81 \%$ & $5 \mathrm{~ms}$ \\
Reversal time & $50 \mathrm{~ns}$ & \\
\end{tabular}

Pattern shape is different as the light parts of the LED pattern are composed of small circular fields which do not reach each other; the Chb pattern consists of illuminated squares that meet at corners.

Total stimulus field is larger in the Chb pattern, the visual angle being twice that occupied by the LED pattern.

Luminance is much higher in the Chb pattern, with a higher value in the middle of the screen than at the periphery.

The illuminated part of the pattern is less than $50 \%$ of the total field in the LED pattern because of the circular design of each diode and the impossibility of placing the diodes edge to edge without intervening space.

Reversal time is a few milliseconds for the Chb pattern because of the movement of the mirror, but is negligible in the LED display.

Recordings were made between two scalp electrodes in EEG positions $\mathrm{Oz}$ and $\mathrm{Fz}$ (10-20 system). One hundred responses were amplified (bandwidth: 0.5 amplitude down at 0.1 and $1000 \mathrm{~Hz}$ ), and summated in a Didac 800 averager. The signal was sampled at 800 points and total averager sweep time was $200 \mathrm{~ms}$ (control group) or $280 \mathrm{~ms}$ (patients). The pattern was reversed at intervals of $700 \mathrm{~ms}(1.4 \mathrm{~Hz})$. The subjects fixated a small green mark in the centre of the projection screen or a small green light-emitting diode in the middle of the LED matrix. The rest of the room was darkened. The latency to the peak of the major positive wave was measured to the nearest millisecond.

\section{Results}

NORMAL SUBJECTS

The control group consisted of subjects with normal visual acuity and no history of visual disturbances (hospital staff and patients without known

diseases of the eyes or visual pathways). Results from stimulation of 52 eyes in 26 subjects with the LED pattern and from stimulation of 38 eyes from 19 individuals with the checkerboard pattern are given in Table 2 . In spite of the much higher luminance of the Chb pattern, it was found that the LED display evoked responses with shorter latencies. This trend can also be seen in Fig. 2A, where VER latencies from a subgroup of 14 normal subjects studied with both methods have been plotted; most values fell below the line that indicates equal latency for LED and Chb methods.

Table 2 Results of recordings of VER in normal subjects stimulated with light-emitting diode pattern (LED) and checkerboard pattern (Chb)

\begin{tabular}{lcc}
\hline & $L E D$ & $C h b$ \\
\hline Number of subjects & 26 & 19 \\
Age (yr) & $20-62$ & $21-62$ \\
Latency of positive peak & & \\
$\quad$ mean (ms) & 93.2 & 96.3 \\
SD* (ms) & 4.8 & 4.1 \\
range (ms) & $80-102$ & $88-103$ \\
Upper normal limit (mean + 3SD) (ms) & 107 & 109 \\
Side difference (ms) & $0-6$ & $0-5$ \\
Mean amplitude ( $\mu \mathrm{V})$ & 5.5 & 11.1
\end{tabular}

*Calculated from mean of latency values for left and right eye in each individual.

Differences in amplitude and shape of the responses to the two methods have been observed, and some of them can be seen in Fig. 1. The LED amplitude is almost always lower (about 50\%) than responses to $\mathrm{Chb}$, and the peak of the major positive wave is less sharp, which can sometimes render exact latency measurements difficult. With LED stimulation there is also sometimes a little negative hump at the peak of the positive wave.

LED

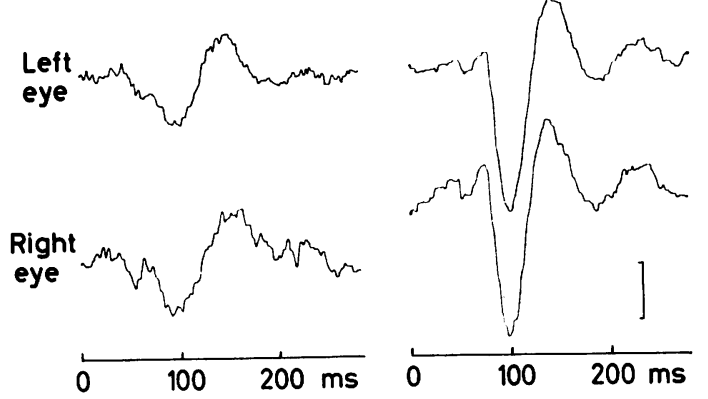

Fig. 1 Recordings from a multiple sclerosis patient showing visual evoked responses to monocular pattern reversal stimulation produced by the LED display and the checkerboard slide projection. Vertical bar $5 \mu \mathrm{V}$. 
The preceding negative peak is usually more clearly developed with Chb stimulation.

There was no significant difference in mean latency between subjects younger than 40 years of age and those above that age.

\section{MULTIPLE SCLEROSIS PATIENTS}

The pattern reversal VER was studied in 38 patients with established or suspected diagnosis of multiple sclerosis based on clinical findings and cerebrospinal fluid examinations. The patients were divided into three diagnostic categories according to the criteria of McAlpine et al. (1972). A definite diagnosis of multiple sclerosis was considered as established in 19 cases, a probable diagnosis in nine patients, and a less certain but possible diagnosis in 10 cases. A history of one or more attacks of retrobulbar neuritis was reported in eight patients in the definite group and six patients in the probable group. In the possible group five cases had a slowly progressive paraplegia without other symptoms.

Patients with a history of visual disturbances such as strabismic amblyopia (Wanger and Nilsson, 1978) or cataract were not included in the study.

In Fig. 2 B-D the latencies of responses to stimulation with the two methods in the three patient groups are shown and can be compared with responses in the normal group shown in Fig. 2A. The number of delayed responses (latency above mean +3 SD) is listed in Table 3 , which also shows the number of cases where no response from one or both eyes could be observed. Such an absence of response was found monocularly in five cases and bilaterally in four cases with the LED stimulus, and in three and two cases respectively with the Chb pattern. If a delayed response or an absence of response in at least one eye is considered as abnormal, a pathological response was found in between $79 \%$ and $90 \%$ of patients with definite or probable multiple sclerosis. In these patients there was no significant difference between the two stimulus methods in their ability to evoke a pathological response. However, in patients with only slight multifocal symptoms or with a progressive paraplegia exclusively (possible multiple sclerosis), a greater frequency of abnormal responses was seen with the LED pattern than with the Chb pattern.

The delays in latency were much greater in the definite and probable multiple sclerosis groups (Fig. 2B and C) than in the possible multiple
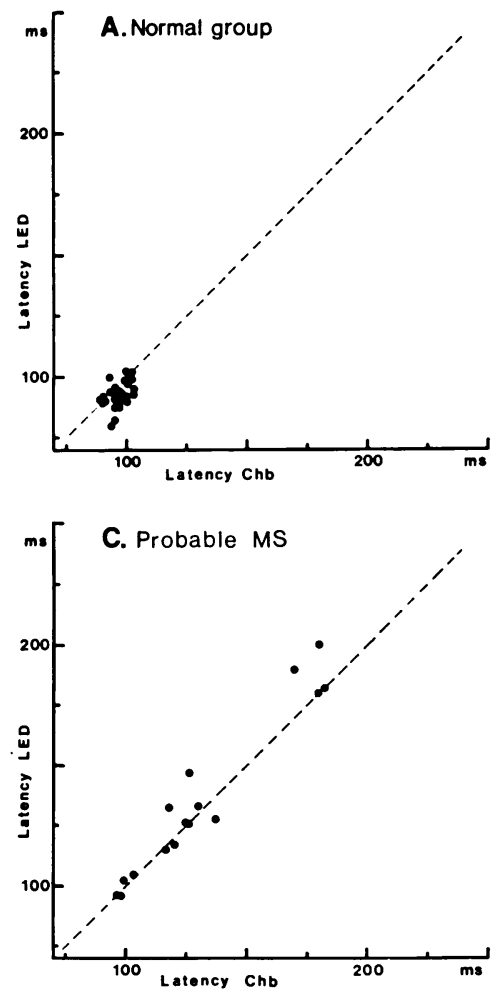
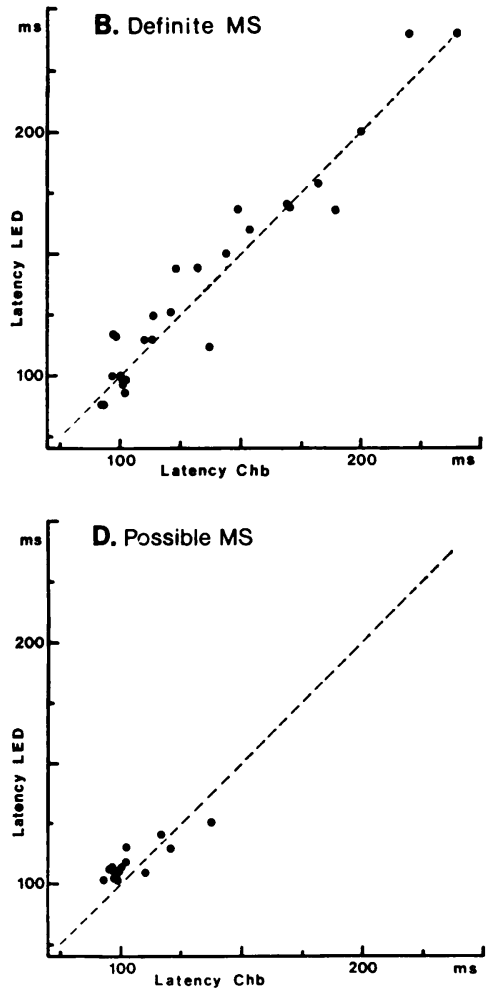

Fig. 2 Latencies to the peak of the major positive wave of the visual evoked responses to LED display and checkerboard (Chb) pattern in a normal control group $(A)$ and in patients with a diagnosis of definite $(B)$, probable $(C)$, or possible $(D)$ multiple sclerosis. Each point represents latency values for one eye. Broken line indicates equal latency with both stimulus methods. 
Table 3 Results of VER recordings in 38 multiple sclerosis patients stimulated with $L E D$ pattern reversal (LED) and checkerboard pattern reversal (Chb)

\begin{tabular}{|c|c|c|c|c|c|c|}
\hline & \multicolumn{6}{|c|}{ Multiple sclerosis diagnosis category } \\
\hline & \multicolumn{2}{|c|}{ Definite } & \multicolumn{2}{|c|}{ Probable } & \multicolumn{2}{|c|}{ Possible } \\
\hline & $L E D$ & $C h b$ & $L E D$ & $C h b$ & $L E D$ & $C h b$ \\
\hline Both eyes normal & 3 & 4 & 1 & 1 & 3 & 7 \\
\hline $\begin{array}{l}\text { one eye delayed } \\
\text { One eye normal, no } \\
\text { response from }\end{array}$ & 1 & 2 & 2 & 2 & 4 & 1 \\
\hline other eye & 1 & 1 & - & - & 1 & - \\
\hline $\begin{array}{l}\text { Both eyes delayed } \\
\text { One eye delayed, no } \\
\text { response from }\end{array}$ & 10 & 9 & 4 & 5 & 一 & 2 \\
\hline $\begin{array}{l}\text { other eye } \\
\text { No response from }\end{array}$ & 1 & 1 & 2 & 1 & 1 & - \\
\hline either eye & 3 & 2 & - & - & 1 & - \\
\hline Total number of patients & \multicolumn{2}{|c|}{19} & \multicolumn{2}{|l|}{9} & \multicolumn{2}{|c|}{10} \\
\hline $\begin{array}{l}\% \text { patients with } \\
\text { pathological }{ }^{*} \text { response }\end{array}$ & & 79 & 90 & 90 & 70 & 30 \\
\hline
\end{tabular}

*Delayed response or absence of response in at least one eye.

sclerosis group (Fig. 2D) where most values lay just below or just above the upper normal limit. A side difference in latency above $6 \mathrm{~ms}$ was not seen in any of the patients with normal latencies for both eyes.

The latencies obtained with the LED pattern can be directly compared with latencies of $\mathrm{Chb}$ responses in Fig. 2. As mentioned above, the values for the control group fell below the line indicating equal latencies, which means that $\mathrm{Chb}$ pattern in almost all normal subjects produced slightly longer latencies than the LED pattern. In the patient groups with definite and probable multiple sclerosis this difference in latency was minimal. However, in the possible multiple sclerosis group most points were situated above the line of equal latencies, which means that for most of the eyes the LED pattern responses showed a slightly longer latency than responses to Chb pattern. As many of these responses had a latency in the vicinity of the upper normal limit, this partly explains why a higher percentage of pathological responses were found with the LED stimulus.

Fourteen patients had a history of retrobulbar neuritis. Thirteen of them had pathological responses with both stimulus methods, and only one patient showed normal responses with both methods.

Five patients among the possible multiple sclerosis cases had a progressive paraplegia as the only symptom. Two of these had normal responses with both stimulus methods, one patient showed prolonged latencies to both types of stimulus, and in two cases LED stimulus responses were found to be delayed or absent while Chb pattern responses were normal.

\section{Discussion}

Both types of pattern reversal stimulus employed in this study produced reproducible VERs in all subjects of a normal control group. The mean normal latency to checkerboard stimulus was less than that found by Halliday et al. (1973) and Matthews et al. (1977) who used a similar presentation technique but with a lower luminance of the white squares. The present mean latency value was, on the other hand, longer than that of Asselman et al. (1975), their pattern being of higher brightness. These differences stress the need for each laboratory to collect its own normal reference values. In spite of the lower luminosity, the LED display produced VER with a shorter mean latency than the Chb stimulus. This must be because of the delay of $5 \mathrm{~ms}$ caused by the slew rate of the moving mirror. However, as the mean difference is about $3 \mathrm{~ms}$ the higher luminance partly compensates for this delay. The lower amplitude and the less sharp peak of the response to LED pattern may also be a result of the lower brightness of diodes, but the smaller total stimulus field may be an additional factor.

The Chb pattern is a more efficient stimulus than the LED display, and this makes it possible in some patients to distinguish responses (mostly with prolonged latencies) to stimulation of eyes that were unresponsive to the LED pattern. The less noisy records and the more clearly defined peaks render latency determinations in $\mathrm{Chb}$ responses easier and more reliable. As a latency delay is a more specific sign of slow conduction in demyelinated nerve fibres than absence of response, the checkerboard pattern reversal used in this study is a more suitable method for diagnosis of multiple sclerosis, especially in cases with a low visual acuity.

The proportion of delayed or absent responses to Chb stimulus seen in multiple sclerosis patients is in good agreement with previous studies using similar stimulators. Thus, among others, Asselman et al. (1975) found a total incidence of $69 \%$, and $84 \%$ among definite cases, and Matthews et al. (1977) reported an overall frequency of $68 \%$, and $75 \%$ in the definite category. Halliday et al. (1973) and Chain et al. (1977) have found still higher proportions of abnormal responses. Similar rates of pathological response have been found when the checkerboard pattern was presented on a television screen (Hennerici et al., 1977): $81 \%$ in definite 
cases and $61 \%$ in all patients with multiple sclerosis.

In the subgroup of cases with possible multiple sclerosis according to the definition by McAlpine et al. (1972), a lower rate of abnormal response to Chb stimulation has been found. The present value of $30 \%$ is similar to frequencies reported by Asselman et al. (1975), Matthews et al. (1977), and Hennerici et al. (1977). Also in this type of patient Halliday et al. (1973) and Chain et al. (1977) described more frequent abnormal responses.

The lower incidence of abnormal VER recordings in the possible multiple sclerosis cases may be due to a low frequency of patients that really do have multiple sclerosis, or to the fact that a demyelination of visual pathways is less common among these patients in comparison with more advanced cases. Another explanation may be that the technique of Chb pattern reversal is not sensitive enough. As the diagnostic value is greatest in this patient category (Hennerici et al., 1977; Matthews et al., 1977), this aspect calls for further refinement of the VER method. New variations in stimulus technique should be compared with results obtained with conventional Chb stimulus in the same patients. In this study a considerably higher incidence of abnormal responses was found when the LED stimulus was used, even if the observed delays were small. This distinction may be due to some difference in the physical properties of the two patterns (see Table 1). For instance, the smaller total field in the LED display restricts the stimulus to the fovea and the immediate parafoveal parts of the retina. Central scotomas are common in multiple sclerosis, which suggests that nerve fibres coming from this area are more easily damaged by a sclerotic plaque than peripheral fibres. This implies that a normal response from the more peripheral area can hide a delayed foveal - parafoveal response. This explanation for the higher sensitivity of the LED pattern is supported by studies by Hennerici et al. (1977) who compared conventional Chb pattern reversal of $20^{\circ}$ field size with responses to the appearance of a small white square subtending $45^{\prime}$ in the centre of the screen. They found that the latter stimulus technique increased the diagnostic yield of the VER method especially in the possible multiple sclerosis category, where the incidence of abnormal responses increased from $43 \%$ to $78 \%$, which corresponds to the increase in sensitivity found in this study when LED stimulation was compared to Chb stimulation (from $30 \%$ to $70 \%$ pathological responses).

Animal studies have shown that information about colour and luminance are transmitted along separate channels and that the optic nerve fibres involved are of different sizes (Gouras, 1968). Certain components in the flash VER show longer latencies when red light is compared to green or yellow light (Cigánek and Ingvar, 1969). The defect in colour vision of ten observed as a sequela to attacks of retrobulbar neuritis even when full visual acuity is regained suggests that the colour channel fibres are more susceptible to demyelination than fibres responding to luminance changes. The red colour of the light-emitting diodes might be of importance in this context. It was recently shown in a study of flash-elicited VER that the delay seen in multiple sclerosis patients was more pronounced with red flash than with white or blue flash (Paty et al., 1976). There is thus clinical as well as neurophysiological support for the concept that colour channels are easily damaged, and this may be the reason for the increased percentage of abnormal responses revealed by a chromatic pattern reversal in cases with early and slight symptoms. McInnes (1977) has also described differences in responses when a black and white checkerboard was compared with a red and white checkerboard pattern, although the differences were more a change in potential shape than a latency prolongation.

Although it is usually easier to get reliable VER with a well-developed positive peak when a reversal of a checkerboard pattern of high luminance is used, it seems as if some properties of the black and red pattern produced by the matrix of low-luminance light-emitting diodes make it more suitable for disclosing evidence of a disturbed impulse transmission in some cases with a minor damage to the visual system. Further studies are necessary to elucidate the factors responsible for this sensitising effect.

I am grateful to $\mathrm{Mr}$ Lars Haapanen, research engineer, who built the LED display and function generator so skilfully.

\section{References}

Arden, G. B., Faulkner, D. J., and Mair, C. (1977). A versatile television pattern generator for visual evoked potentials. In Visual Evoked Potentials in Man, pp. 90-109. Edited by J. E. Desmedt. Clarendon Press: Oxford.

Asselman, P., Chadwick, D. W., and Marsden, C. D. (1975). Visual evoked responses in the diagnosis and management of patients suspected of multiple sclerosis. Brain, 98, 261-282.

Bornstein, Y. (1975). The pattern evoked responses (VER) in optic neuritis. Albrecht v Graefes Archiv für Klinische und Experimentelle Ophthalmologie, 197, 101-106. 
Bynke, H., Elmqvist, D., and Rosén, I. (1977). Visual evoked responses to pattern reversal stimulation. Electroencephalography and Clinical Neurophysiology, 43, 442.

Chain, F., Mallecourt, J., Leblanc, M., and Lhermitte, F. (1977). Apport de l'enregistrement des potentiels évoqués visuels au diagnostic de la sclérose en plaques. Revue Neurologique, 133, 81-88.

Cigánek, L., and Ingvar, D. H. (1969). Colour specific features of visual cortical responses in man evoked by monochromatic flashes. Acta Physiologica Scandinavica, 76, 82-92.

Evans, B. T., Binnie, C. D., and Lloyd, D. S. L. (1974). A simple visual pattern stimulator. Electroencephalography and Clinical Neurophysiology, 37, 403-406.

Feinsod, M., and Hoyt, W. F. (1975). Subclinical optic neuropathy in multiple sclerosis. Journal of Neurology, Neurosurgery, and Psychiatry, 38, 1109-1114.

Gouras, P. (1968). Identification of cone mechanisms in monkey ganglion cells. Journal of Physiology, 199, 533-547.

Halliday, A. M., McDonald, W. I., and Mushin, J. (1972). Delayed visual evoked response in optic neuritis. Lancet, 1, 982-985.

Halliday, A. M., McDonald, W. I., and Mushin, J. (1973). Visual evoked response in diagnosis of multiple sclerosis. British Medical Journal, 4, 661-664.

Hennerici, M., Wenzel, D., and Freund, H.-J. (1977). The comparison of small-sized rectangle and checkerboard stimulation for the evaluation of delayed visual evoked responses in patients suspected of multiple sclerosis. Brain, 100, 119-136.
McAlpine, D., Lumsden, C. E., and Acheson, E. D. (1972). Multiple Sclerosis. A Reappraisal. Livingstone: Edinburgh and London.

McInnes, A. (1977). The visual evoked responses to a red and white checkerboard pattern in patients with suspected multiple sclerosis. Electroencephalography and Clinical Neurophysiology, 43, 286.

Matthews, W. B., Small, D. G., Small, M., and Pountney, E. (1977). Pattern reversal evoked visual potential in the diagnosis of multiple sclerosis. Journal of Neurology, Neurosurgery, and Psychiatry, 40, $1009-1014$.

Nilsson, B. Y. (1977). Visual evoked response elicited by pattern-reversal stimulation. Electroencephalography and Clinical Neurophysiology, 43, 442-443.

Paty, J., Brenot, Ph., Henry, P., and Faure, J. M. A. (1976). Potentiels évoqués visuels et sclérose en plaque. Revue Neurologique, 132, 605-621.

Purves, S. J., and Low, M. D. (1976). Visual evoked potentials to a reversing-pattern light-emitting diode stimulator in normal subjects and patients with demyelinating disease. Electroencephalography and Clinical Neurophysiology, 41, 651-652.

Regan, D., Milner, B. A., and Heron, J. R. (1976). Delayed visual perception and delayed visual evoked potentials in the spinal form of multiple sclerosis and in retrobulbar neuritis. Brain, 99, 43-66.

Wanger, P., and Nilsson, B. Y. (1978). Visual evoked responses to pattern-reversal stimulation in patients with amblyopia and/or defective binocular functions. Acta Ophthalmologica Scandinavica. In press. 